\title{
Phage typing of Salmonella Typhimurium - is it still a useful tool for surveillance and outbreak investigation?
}

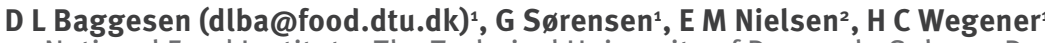

1. National Food Institute, The Technical University of Denmark, Søborg, Denmark

2. Statens Seruminstitut, Copenhagen S, Denmark

Citation style for this article:

Citation style for this article: Baggesen DL, Sørensen G, Nielsen EM, Wegener HC. Phage typing of Salmonella Typhimurium - is it still a useful tool for surveillance and outbreak investigation?. Euro Surveill. 2010;15(4):pii=19471. Available online: http://www.eurosurveillance.org/ViewArticle.aspx?Articleld=19471

This article has been published on 28 January 2010

Phage typing has for decades been useful as a phenotypical, definitive method for epidemiological characterisation of Salmonella Typhimurium. The system recommended by the World Health Organization (WHO) Collaborative Centre for phage typing of Salmonella has, however, become rather complex, and the present study illustrates the challenges of sufficient standardisation of the interpretation of lysis results to make sure that the same strain is assigned to the same phage type in different laboratories. Even though molecular typing methods will replace phenotypic characterisation methods in the future, it is our opinion that phage typing will remain for some time a useful tool to strengthen global Salmonella surveillance. Therefore, improved standardisation and quality assurance is essential to obtain a robust and harmonised method that allows comparison of results between laboratories.

Epidemiological characterisation of pathogens causing human outbreaks of food-borne disease is essential for many reasons. The outbreak is often identified by increased registration of a specific pathogen (the Epi-type) during routine diagnostics and surveillance, and is further confirmed by detailed molecular characterisations that support the hypothesis of a common source of origin. Furthermore, identification of similar strains in historical specimen collections from monitoring programmes of food and food animal production can contribute to the generation of a hypothesis for the outbreak investigation. During the outbreak investigation, epidemiological characterisation constitutes the basis for the comparison of strains from human cases and potential sources, and finally, when the actual food source is found, constitutes the final demonstration of the infection source.

In the last decades more and more food-associated outbreaks have involved more than one country and even more than one continent, primarily due to the ever increasing globalisation of the food supply [1-3]. Identification and control of food-borne disease outbreaks of international significance can only be performed when professionals work together and agree on common methods applicable for definitive characterisation of pathogens like, in our case, Salmonella.

Serotyping according to the Kauffmann-White scheme [4] has for more that 80 years been the primary characterisation of Salmonella. This method is widely applied all over the world and harmonised to a degree that allows results to be compared between laboratories and countries. Some serovars, e.g. Salmonella Enteritidis and S. Typhimurium, are, however, so dominant in especially Europe and the United States that further characterisation is needed for surveillance.

In Denmark, phage typing as described by the World Health Organization (WHO) Collaborative Centre for phage typing of Salmonella (Health Protection Agency (HPA), Colindale, United Kingdom) has been applied for surveillance of $S$. Enteritidis and $S$. Typhimurium in humans, food and food production animals. Phage typing has proven to be an important tool for strain characterisation and the results obtained have been used since the mid-9os in surveillance, source attribution and outbreak investigations [5,6]. Phage typing is, however, also a phenotypical method that depends very much on the experience of the individual laboratory and on support from the reference centre that coordinates the maintenance of phages and the updating of the system. Only when the phage typing method is harmonised and the performance in different laboratories is controlled, can the results be regarded as definitive and comparable between laboratories.

The challenges encountered when using phage typing have become clear in a number of outbreak investigations in Denmark. A year after a large outbreak of human salmonellosis in April 2008 [7], more than 1,300 cases have been registered, and despite intensive epidemiological and microbiological investigations it has not been possible to identify the infection source. The outbreak had been identified due to an increase in the number of laboratory registrations of human $S$. Typhimurium with a unique multiple-locus variablenumber tandem repeat (VNTR) analysis (MLVA) type corresponding to phage type U292. The strains were 
further characterised by pulsed-field gel electrophoresis (PFGE) which showed a unique Xbal-profile, confirming the epidemiological relationship between the human cases [8].

The strains from the outbreak described here were phage-typed at the National Food Institute as is routine for all $S$. Typhimurium strains from human cases, food items and food productions animals in Denmark. The lysis pattern obtained from the initial typing was characterised by strong reactions with phages 11 and 14 , and weaker reactions with phages 26 and 35 , although some variations in the extent of lysis were observed during the outbreak that did not influence the phage type. There was no reaction with the remaining routinely used phages. This lysis pattern was in acceptable agreement with phage type U292. In summer 2008 during the continued outbreak, representative outbreak strains were sent to the WHO Collaborative Centre for phage typing of Salmonella, where they were characterised as $\mathrm{U}_{276}$. The results obtained there were similar to those obtained at the National Food Institute, but the interpretation of the results was different. Identical strains from this outbreak were therefore assigned to two different phage types by two experienced laboratories. As the different interpretation was the result of weighing the interpretation of all lysis reactions against the others, it can be difficult to judge which is the 'right' type designation.

Even though phage typing during this outbreak has been a valuable tool for the national investigation, the disagreement between two laboratories puts into question its usefulness as a definitive typing system that would allow comparison and communication between laboratories and countries. Disagreement between laboratories has caused confusion in past outbreak investigations: In 2003, an outbreak of human salmonellosis was identified in Sweden, and Danish pork was pointed out as the most probable source of infection. The source of infection was identified as S. Typhimurium DT108, but because this Salmonella type had not been detected by the Danish surveillance of pigs and pork production, pork did not seem to be the likely source. However, based on further joint investigation by the two countries it emerged that the strain initially identified as S. Typhimurium DT108 in Sweden was known as $S$. Typhimurium DT170 in the Danish surveillance, and consequently Danish pork could not be ruled out as a probable source (unpublished results, Baggesen, 2003). Late in 2008, an outbreak of $S$. Typhimurium characterised by a specific MLVA-type was identified in Sweden, Norway and Denmark and traced back to Danish pork. Strains related to this outbreak was assigned to several phage types (U288 in Denmark, RDNC in Norway and $\mathrm{U}_{302}$ in Sweden), and only due to inclusion of MLVA results, these cases were recognised as a common outbreak [9].

Should these and similar experiences disqualify phage typing as method for definitive strain characterisation in relation to diagnostics and surveillance of Salmonella infections? Several authors have suggested that genotyping methods such as PFGE and MLVA, combined with harmonised computer-based evaluation of the typing results and electronic exchange of data, can fulfil the requirements for definitive typing $[10,11]$.

There is no doubt that molecular typing methods, eventually whole genome sequencing, will replace phenotypic characterisation methods in the future. But is this time now? Compared with the genotyping methods, sero- and phage typing are cheap and less labour-intensive methods based on simple technology for which only limited equipment is needed. This opens an opportunity for screening a large number of Salmonella strains as part of human diagnostics and monitoring programmes in food and food production animals not only in the developed part of the world but also in developing countries. Nowadays, more and more countries contribute to the international food supply. A strengthening of the global Salmonella surveillance with improved characterisation of strains from humans, food and animals, and sharing of the results among professionals is essential for food safety.

The outbreaks described in this paper suggest that phage typing currently has limitations, which could become worse if it was to be implemented globally for Salmonella surveillance. Improved standardisation and quality assurance is essential in order to obtain a robust and harmonised method that allows comparison of results between laboratories. The phage typing system recommended by the WHO Collaborative Centre and applied in the studies described here was first described 1959 by Callow [12] and has since been extended. Today it utilises a comprehensive number of phages, which leads to a large number of different patterns and thereby phage types. Assignment of a lysis pattern to a specific phage type is based on interpretation of the individual lysis reaction and comparison to a standard scheme of lysis patterns and phage types, a procedure that leaves room for conflicting results.

In addition, experience from the WHO Global SalmSurv programme (http://www.who.int/salmsurv/en) has proven that the capacity and quality of Salmonella serotyping can be enhanced through regular training of diagnostics staff and implementation of international external quality assurance systems [13]. It is likely that similar capacity-building and harmonisation efforts could improve phage typing.

We believe that phage typing can, for a while yet, play an important role in surveillance and control of the common Salmonella serotypes. However, this requires strengthened efforts to make the system available to more laboratories internationally, possibly a simplification of the system to enhance its robustness even though this may slightly compromise its discriminatory power, and finally improved external and internal quality assurance systems. 


\section{References}

1. Aarestrup FM, Hendriksen RS, Lockett J, Gay K, Teates K, McDermott PF, et al. International spread of multidrug resistant Salmonella Schwarzengrund in food products. Emerg Infect Dis. 2007;13(5):726-31.

2. Nygård K, Lassen J, Vold L, Andersson Y, Fisher I, Löfdahl $S$, et al. Outbreak of Salmonella Thompson Infections Linked to Imported Rucola Lettuce. Foodborne Pathog Dis. 2008;5(2):165-73.

3. Pezzoli L, Elson R, Little CL, Yip H, Fisher I, Yishai R, et al. Packed with Salmonella-Investigation of an Internationa Outbreak of Salmonella Senftenberg Infection Linked to Contamination of Prepacked Basil in 2007. Foodborne Pathog Dis. 2008;5(5):661-8.

4. Grimont PAD, Weill F-X.ANTIGENIC FORMULAE OF THE SALMONELLA SEROVARS. 9th ed. Paris: WHO Collaborating Centre for Reference and Research on Salmonella, Institut Pasteur. 2007.

5. Baggesen DL, Wegener HC. Phage types of Salmonella enterica ssp. enterica serovar typhimurium isolated from production animals and humans in Denmark. Acta Vet Scand. 1994;35(4):349-54.

6. Hald T, Lo Fo Wong DM, Aarestrup FM. The Attribution of Human Infections with Antimicrobial Resistant Salmonella Bacteria in Denmark to Sources of Animal Origin. Foodborne Pathog Dis. 2007;4(3):313-26

7. Ethelberg S, Wingstrand A, Jensen T, Sørensen G, Müller L, Lisby M, et al. Large outbreaks of Salmonella Typhimurium infection in Denmark in 2008. Euro Surveill. 2008;13(44):pii=19023. Available from: http://www. eurosurveillance.org/ViewArticle.aspx?Articleld=19023

8. Ethelberg S, Wingstrand A, Jensen T, Sørensen G, Müller L, Nielsen EM, et al. Large ongoing outbreak of infection with Salmonella Typhimurium U292 in Denmark, February July 2008. Euro Surveill. 2008;13(28):pii=18923. Available from: http://www.eurosurveillance.org/ViewArticle. aspx?Articleld $=18923$

9. Bruun T, Sørensen G, Forshell LP, Jensen T, Nygard K, Kapperud G, et al. An outbreak of Salmonella Typhimurium infections in Denmark, Norway and Sweden, 2008. Euro Surveill. 2009;14(10):pii=19147. Available from: http://www. eurosurveillance.org/ViewArticle.aspx?Articleld=19147

10. Torpdahl M, Sørensen G, Lindstedt B-A, Nielsen EM. Tandem Repeat Analysis for Surveillance of Human Salmonella Typhimurium Infections. Emerg Infect Dis. 2007;13(3):388-95

11. Gatto AJ, Peters TM, Green J, Fisher IS, Gill ON, O’brien SJ, et al. Distribution of molecular subtypes within Salmonella enterica serotype Enteritidis phage type 4 and S. Typhimurium definitive phage type 104 in nine European countries, 20002004: results of an international multi-centre study. Epidemiol Infect. 2006;134(4):729-36.

12. Callow BR. A new phage-typing scheme for Salmonella typhimurium. J Hyg (Lond). 1959;57:346-59

13. Hendriksen RS, Mikoleit M, Carlson VP, Karlsmose S, Vieira $A R$, Jensen $A B$, et al. WHO Global Salm-Surv External Quality Assurance System (EQAS) for serotyping of Salmonella isolates, 2000-2007. J Clin Microbiol. 2009;47(9):2729-36. 